\title{
Caracterización de cerámicos inmersos en soluciones del sistema sio2 - tio2 - zro2 sintetizadas por el método sol-gel
}

\section{Characterization of ceramics, immersed in solutions of the system sio2 - tio2 - zro2 synthesized by the sol-gel method}

\author{
Sandra Rozo-Rincón ${ }^{1}$, Jhon Gelves-Díaz ${ }^{2}$, Jorge Sánchez-Molina ${ }^{3}$, Jorge Bautista-Ruiz ${ }^{4}$ \\ ${ }^{1}$ Universidad Francisco de Paula Santander, Colombia. Email: sandrarozo85@ @otmail.com \\ ${ }^{2}$ Grupo de Investigación en Competitividad y Sostenibilidad para el desarrollo, Universidad Libre sede Cúcuta, Colombia. \\ Email: johngelves@yahoo.es \\ ${ }^{3}$ Centro de Investigación de Materiales Cerámicos - CIMAC, Universidad Francisco de Paula Santander, Colombia. \\ Email: jorgesm@ufps.edu.co \\ ${ }^{4}$ Grupo de Investigación en Tecnología Cerámica - GITEC, Universidad Francisco de Paula Santander, Colombia. \\ Email: jorgebautista@ufps.edu.co
}

RECIBIDO: Enero 19, 2017. ACEPTADO: Marzo 20, 2017. VERSIÓn FInAL: Mayo 30, 2017.

\begin{abstract}
RESUMEN
Se presentan los resultados de la caracterización físico-cerámica, microquímica y estructural de muestras cerámicas de arcilla roja inmersas en soluciones de $\mathrm{SiO} 2$ - $\mathrm{TiO} 2$ - $\mathrm{ZrO} 2$ obtenidas por el método Sol-Gel utilizando tetraetil ortosilacato, tetrabutóxido de titanio y tetrabutóxido de zirconio. Las muestras cerámicas fueron elaboradas con polvos atomizados de arcilla roja, cocidas a $1000^{\circ} \mathrm{C}$. Después de la cocción las cerámicas fueron inmersas en las soluciones en concentraciones en volumen 10/20/70: $\mathrm{Si} / \mathrm{Ti} / \mathrm{Zr}$ y 10/70/20: $\mathrm{Si} / \mathrm{Ti} / \mathrm{Zr}$ con tiempos de permanencia de 2 , 4 y 6 minutos. Posterior al secado las muestras se trataron térmicamente a $600^{\circ} \mathrm{C}$ durante 60 minutos, a una velocidad de $2^{\circ} \mathrm{C} / \mathrm{min}$. Aspectos microquímicos y microestructurales de las muestras cocidas fueron establecidos mediante el uso de difracción de rayos X (DRX), espectroscopia de infrarrojo (FTIR) y detección de energías dispersivas de rayos X (EDX). Las propiedades tecnológicas evaluadas fueron la absorción de agua, densidad aparente y resistencia mecánica a la flexión. El estudio permitió establecer que las muestras inmersas en la solución 10/70/20: $\mathrm{Si} / \mathrm{Ti} / \mathrm{Zr}$ permiten incrementar la resistencia mecánica a la flexión. De igual manera se observó una ligera reducción del porcentaje de absorción de agua con este mismo tratamiento. El tratamiento con mayor presencia de zirconio no muestra cambios significativos respecto al patrón empleado (muestra sin inmersión).
\end{abstract}

PALABRAS ClAVE: Arcilla roja, Cerámicos, Resistencia mecánica a la flexión, Sistema SiO2 - TiO2 - ZrO2, Solgel.

\begin{abstract}
The results of structural, microchemical and physicoceramic characterization of ceramic samples (red clay) immersed in solutions "TiO2 - $\mathrm{ZrO} 2-\mathrm{SiO}_{2}$ are presented. Samples were prepared by the sol-gel method using tetraethyl ortosilicate, titanium tetrabutoxide and zirconium tetrabutoxide. Ceramics samples for immersion were prepared with atomized powders of red clay and subjected to firing at $1000{ }^{\circ} \mathrm{C}$. The samples were made from ceramic powders atomized red clay, fired at $1000^{\circ} \mathrm{C}$. After firing ceramics were immersed in the solutions in volume concentrations 10/20/70: $\mathrm{Si} / \mathrm{Ti} / \mathrm{Zr}$ and 10/70/20: $\mathrm{Si} / \mathrm{Ti} / \mathrm{Zr}$. Immersion times were 2, 4 and 6 minutes respectively. Subsequent to drying, the samples were subjected to firing at $600{ }^{\circ} \mathrm{C}$ for 1 hour, at a rate of $2{ }^{\circ} \mathrm{C} / \mathrm{min}$. Microchemical and
\end{abstract}

Este artículo puede compartirse bajo la licencia CC BY-ND 4.0 y se referencia usando el siguiente formato: S.M Rozo Rincón, J.F Gelves Díaz, J. Sánchez Molina, J.H Bautista Ruiz, "Caracterización de cerámicos inmersos en soluciones del sistema sio2 - tio2 - zro2 sintetizadas por el método sol-gel”, UIS Ingenierías, vol. 16, no. 2, pp. 51-60, Julio-Diciembre 2017. Doi: https://doi.org/10.18273/revuin.v16n2-2017005 
microstructural aspects of the fired samples were established using X-ray diffraction (XRD), infrared spectroscopy (FTIR) and x-ray dispersive energy (EDX). Technological properties evaluated were water absorption, bulk density and mechanical resistance to flexion. The study results allowed to determine that mechanical resistance to flexion improved in samples immersed in the solution 10/70/20: Si/Ti/Zr. Similarly, a slight reduction in the percentage of water absorption with this same treatment was observed. Treatment with greater amount of zirconium shows no significant changes in relation to the standard used.

KEYWORDS: Red clay, Ceramic, Sol-gel, Mechanical resistance to flexion, $\mathrm{SiO} 2$ - TiO2 - ZrO2 system.

\section{INTRODUCCIÓN}

La industria cerámica de la construcción juega un papel importante en la económica del departamento Norte Santander. Los productos a base de arcilla roja fabricados en la región, como ladrillos, tejas y baldosas son reconocidos en el mercado nacional e internacional [1]. A pesar de ello y de la larga tradición del gremio cerámico en el departamento, han sido pocos los adelantos tecnológicos obtenidos en comparación con los grandes referentes a nivel mundial como lo son España e Italia [2,3]. Las conclusiones de los estudios referenciados resaltan la necesidad de mejorar los aspectos de innovación con mirar de alcanzar una mejor competitividad.

Con el fin de mejorar la innovación del sector cerámico regional se debe dar respuesta a las necesidades tangibles de su sociedad tal como lo han hecho los países referentes [4]. Como ejemplo de innovación destacan la obtención de baldosas y recubrimientos con capacidad de autolimpiado y autodesinfección, baldosas fosforescentes que captan y emiten luz en ausencia de la fuente, baldosas antielectrostáticas que impiden la acumulación de carga eléctrica o baldosas capaces de aprovechar la energía solar [5-7]. Otros aspectos más comunes que se destacan en la literatura son el incremento de la dureza y de la resistencia al ataque químico [8-10]. Muchas de estas características se han obtenido mediante tratamientos especiales entre ellos el uso de partículas nanométricas [11].

Una de las técnicas empleadas para mejorar las propiedades de los sustratos es el Sol-gel. A través de este procedimiento se pueden obtener cerámicos para conformar recubrimientos (películas finas o gruesas) y obtener fibras. Esto ha permitido que el método Sol-gel adquiera un gran interés científico y tecnológico [12-16].

Este trabajo de investigación contempló la inmersión de cerámicos tradicionales (probetas tipo baldosa elaborados a base de arcillas y feldespatos) en soluciones obtenidas por el método Sol - Gel empleando un compuesto del sistema $\mathrm{SiO} 2-\mathrm{TiO} 2-\mathrm{ZrO} 2$. La finalidad del trabajo ha sido la exploración de nuevas rutas para el incremento de la resistencia mecánica de baldosas cerámicas de alta porosidad (>8\%) [17].

\section{METODOLOGÍA}

\subsection{Conformado de muestras cerámicas}

Se conformaron las muestras cerámicas por prensado uniaxial a una presión de 60 Bar. La pasta cerámica empleada es una mezcla de arcilla roja del municipio de El Zulia (con presencia de moscovita), feldespato del municipio de Sardinata y caliza del municipio de Los Patios (todos jurisdicción del Norte de Santander). Las dimensiones de las probetas elaboradas fueron $10 \mathrm{~cm}$. $\mathrm{x}$ $5 \mathrm{~cm}$. x $1 \mathrm{~cm}$. Posterior al conformado las muestras se secaron a temperatura ambiente durante 24 horas, y luego en estufa de secado a $110^{\circ} \mathrm{C}$. El tratamiento térmico de las muestras fue de $1000{ }^{\circ} \mathrm{C}$ durante 1 hora en horno mufla (modelo 10/12 marca Gabbrielli), empleando atmosfera oxidante.

\subsection{Preparación del sol-gel}

Para la síntesis química de los soles, se utilizó el procedimiento establecido en trabajos anteriores $[12,16]$. Se utilizó como precursores el Tetraetil ortosilicato [Si(OC2H6)] precursor del óxido de silicio, Tetrabutoxido de titanio [Ti(OBu)4], precursor del óxido titanio y Tetrabutóxido de zirconio [ $\mathrm{Zr}(\mathrm{OC} 3 \mathrm{H} 7) 4]$, precursor del óxido de zirconio. Adicionalmente se empleó etanol como solvente y 2,4-pentanodiona como acomplejante. Se prepararon dos concentraciones de las soluciones $\mathrm{Si}-\mathrm{Ti}-\mathrm{Zr}$ (10-20-70) y (10-70-20), se dejaron en envejecimiento para favorecer las reacciones de poli-condensación por un tiempo de 45 días, a $28{ }^{\circ} \mathrm{C}$ y humedad relativa del $31 \%$.

\subsection{Tratamiento a las muestras cerámicas}

Las muestras cerámicas fueron inmersas en las soluciones por tiempos de 2, 4 y 6 minutos. Éstas se dejaron secar a temperatura ambiente de $28{ }^{\circ} \mathrm{C}$ durante 4 horas. Posteriormente se sometieron a un proceso de cocción en horno mufla a la temperatura de $600{ }^{\circ} \mathrm{C}$ durante 1 hora, con una velocidad de calentamiento de 2 ${ }^{\circ} \mathrm{C} /$ minuto. 


\subsection{Caracterización}

La caracterización estructural de las muestras cerámicas se desarrolló a través de difracción de rayos X, mediante un difractómetro de polvo marca Rigaku Miniflex 3D/Max., en condiciones de trabajo de Voltaje $40 \mathrm{kV}$, corriente $30 \mathrm{~mA}$, rango de medición entre 3 y $70^{\circ}$ en configuración 2 theta, Radiación CuKa1, Filtro de Níquel, Tipo de barrido paso a paso a $0,2^{\circ}$ y tiempo de conteo por paso de 2 segundos. El patrón de difracción fue comparado con la información presente en la base de datos PDF-2 del International Centre for Diffraction Data (ICDD). Complementariamente espectroscopia de infrarrojo fue usada para caracterizar el material. En este caso en particular fue usada para reconocer la presencia de vibraciones características de fases con presencia de titanio o zirconio. Para ello se empleó un equipo marca Shimadzu, modelo FTIR 8400S y una prensa manual, para el conformado de las pastillas $(1,0 \mathrm{mg}$ de muestra con $100 \mathrm{mg}$ de $\mathrm{KBr}$ ), marca Carver modelo 4350L, aplicando una presión de 10 t. Las condiciones de trabajo fueron: modo transmitancia, rango de barrido entre $400 \mathrm{~cm}-1$ y $4000 \mathrm{~cm}-1$, resolución de $4 \mathrm{~cm}-1$ y número de escaneos igual a 48. El software de trabajo fue el IR Solution versión 1.4.

Los aspectos microquímicos fueron establecidos en un microscopio electrónico de barrido Quanta FEG 650 ambiental (ESEM/EDX), trabajando en el modo de alto vacío. Se realizó el análisis EDX con aceleración de $15 \mathrm{kV}$, el detector utilizado es un Apolo X con resolución de $126 \mathrm{eV}$ (en Mn) y el software génesis para el análisis EDX. Se utilizó un área de análisis de 300 micrómetros de largo y 250 micrómetros de alto.

El análisis de resistencia mecánica a la flexión se llevó a cabo en un equipo de laboratorio Marca Gabbrielli. Con este equipo se tomaron las medidas del módulo de rotura de las muestras cerámicas cocidas, empleando la metodología de la norma técnica colombiana NTC 43214. [17].

Dentro de los ensayos físico - cerámicos de las muestras se determinó la absorción de agua siguiendo el procedimiento descrito en. NTC 4321- 3 [18] y densidad aparente de las muestras por el método de inmersión en mercurio.

\section{RESULTADOS Y DISCUSIÓN}

\subsection{Caracterización Estructural}

Los patrones de difracción por rayos $\mathrm{X}$ de las muestras cerámicas inmersas en las concentraciones $\mathrm{Si} / \mathrm{Ti} / \mathrm{Zr}$ : 10/20/70 y Si/Ti/Zr: 10/70/20 en función de los tiempos de inmersión a 2, 4 y 6 minutos se presentan en las Figuras 1 y 2 , respectivamente.

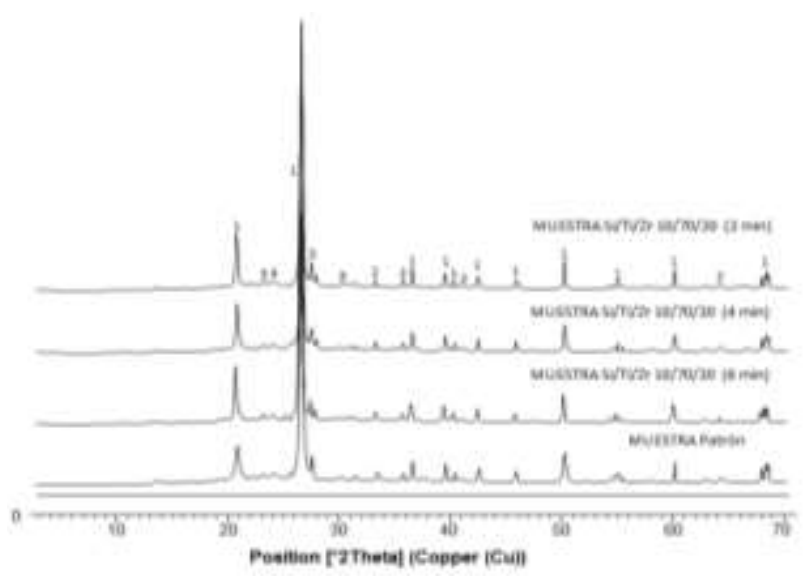

Figura 1. Patrones de difracción para muestras cerámicas inmersas en la concentración $\mathrm{Si} / \mathrm{Ti} / \mathrm{Zr}$ : 10/70/20 a diferentes tiempos. 1: cuarzo; 2: hematita; 3: feldespato potásico.

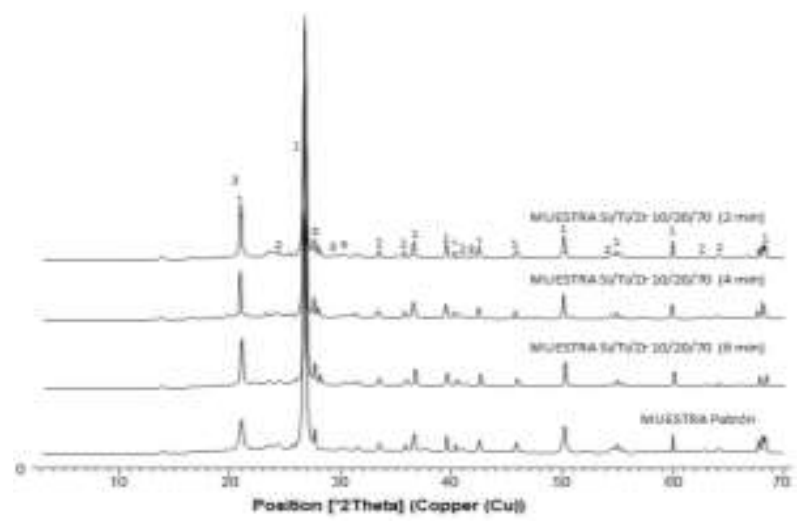

Figura 2. Patrones de difracción para muestras cerámicas inmersas en la concentración Si/Ti/Zr: 10/20/70 a diferentes tiempos.1: cuarzo; 2: hematita; 3: feldespato potásico.

Mediante la caracterización estructural usando DRX se ha podido establecer que luego del proceso de cocción, no se evidencian diferencias significativas entre la muestra patrón (cerámico sin inmersión) y los tratamientos realizados. La fase cristalina mayoritaria en todas las muestras es el cuarzo, seguido por feldespato potásico, feldespatos de la solución sólida de la plagioclasa y hematita. Como fases cristalinas minoritarias, los ajustes de software dejan ver la posible presencia de rutilo, mullita, baddeleyita, rankinita y pigeonita, especialmente en las mezclas inmersas en la solución con mayor presencia de zirconio. También destaca la presencia de fase amorfa (se evidencia la presencia de un ligero abultamiento en la región $2 \theta$ comprendida entre $18^{\circ}$ y $35^{\circ}$ característico de aluminosilicatos amorfos). 
En la Figura 3 se presenta en más detalle los cambios ocasionados en las fases presentes en la pasta cerámica después del proceso de cocción (patrones de difracción).
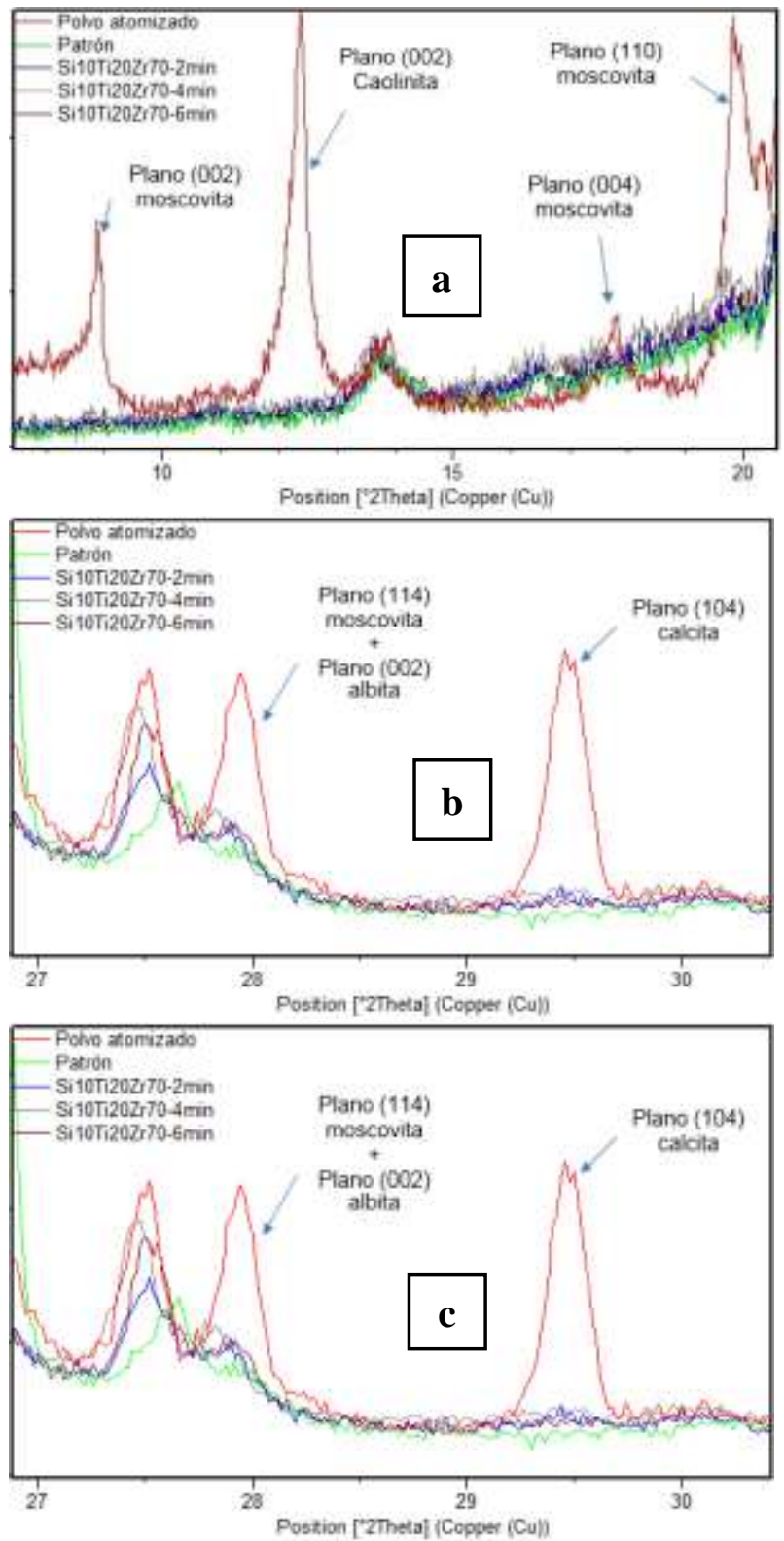

Figura 3. Cambios evidenciados en la pasta cerámica después del proceso de cocción. a) En fases arcillosas; b) Calcita y feldespato tipo albita; c) En hematita.

La Figura 3a muestra los cambios ocasionados sobre las fases arcillosas. La caolinita sufre una pérdida total de cristalinidad, este hecho se evidencia por la desaparición del principal plano de reflexión (001) en la posición $2 \theta$ cercana a $12,4^{\circ}$. En el caso de la moscovita se evidencian reducciones en el plano (002) en la posición $2 \theta$ cercana a $8,9^{\circ}$ y en el plano (110) en la posición $2 \theta$ cercana a $19,8^{\circ}$ tal como se aprecia en la figura 3 a.
Caso similar se da para la calcita presente en el polvo atomizado, debido a la desaparición del plano (104) en la posición $2 \theta$ cercana a $29,6^{\circ}$ evidenciado en la Figura $3 b$, posiblemente debido a la descomposición de la fase carbonatada [19]. La fase feldespática "albita" fue identificada en la pasta cerámica, después del proceso cerámico, se evidenció una reducción en la intensidad de sus planos de reflexión, en la figura $3 \mathrm{~b}$ se aprecia un decrecimiento de la reflexión del plano (002) de esta fase.

Por otro lado se encuentran las fases que han mostrado un incremento en la intensidad de los planos de reflexión, en este caso se encuentra la hematita ( $\mathrm{Fe} 2 \mathrm{O} 3)$. Esta fase fue evidenciada en la pasta cerámica, sin embargo después del proceso de cocción se observó un aparente aumento en su concentración, este hecho es soportado por un incremento de la reflexión del plano (004) en la posición $2 \theta$ cercana a $33,2^{\circ}$ evidenciado en la Figura $3 \mathrm{c}$. Dicho incremento en la fase podría ser causado por la oxidación del hierro proveniente de la descomposición de la moscovita presente en la pasta cerámica.

En la Figura 4 se ha resaltado los cambios causados por la inmersión de las muestras cocidas en las soluciones del sistema $\mathrm{SiO} 2$ - $\mathrm{TiO} 2$ - $\mathrm{ZrO} 2$. En la Figura 4a se aprecia un incremento en la intensidad de plano (101) de la fase cuarzo (respecto a la muestra patrón) en la posición $2 \theta$ cercana a $26,6^{\circ}$ para todos los tratamientos de inmersión. Este hecho podría deberse a una posible formación de la fase cuarzo causado por la oxidación del silicio proveniente de la solución de inmersión.

En relación a la adición de titanio, producto de la inmersión de las muestras en la solución del sistema $\mathrm{SiO} 2$ - TiO2 - ZrO2, se evidenció mediante difracción de rayos $\mathrm{X}$, la formación de la fase rutilo en todos los tratamientos realizados. Su concentración es baja, apenas identificable mediante la técnica de DRX. En la Figura $4 \mathrm{~b}$ se pueden apreciar los cambios presentes en el plano de mayor reflexión de la fase rutilo (211). Se evidencia un ligero incremento de la señal de dicho plano en las muestras sometidas a inmersión, lo cual podría sugerir una mayor concentración de la fase después de realizada la cocción. Asumiendo que no hay efectos de orientación preferencial en los cristales y que existe una relación directa entre la concentración de la fase con la intensidad de los planos de reflexión, se puede establecer el siguiente orden de concentración de la fase: patrón< Si10-Ti70-Zr20-2 min< Si10-Ti70-Zr20-4 min= Si10Ti70-Zr20-6 min. Sin embargo, esta tendencia cambia para la solución con mayor presencia de zirconio el orden obtenido es el siguiente: patrón< Si10-Ti70-Zr20-2 $\min =$ Si10-Ti70-Zr20-6 min<Si10-Ti70-Zr20-4 min, con 
lo cual se puede inferir que el tiempo de inmersión "si" tiene un efecto en la formación de la fase rutilo en la muestra cerámica.
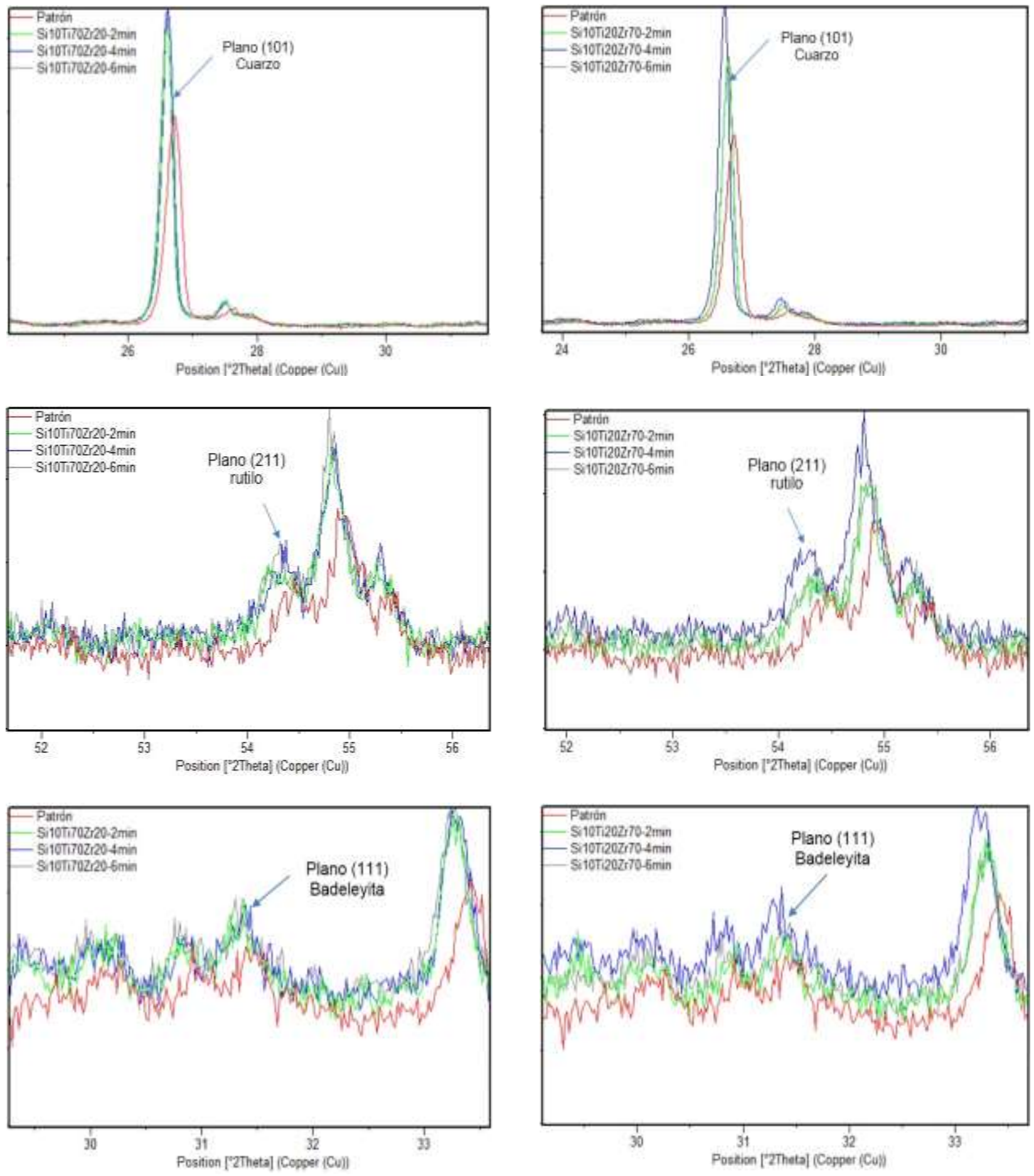

Figura 4. Cambios evidenciados en las muestras inmersas en el sistema $\mathrm{SiO} 2$ - TiO2 - ZrO2 después del proceso de cocción a $600{ }^{\circ} \mathrm{C}$. 
En relación a la presencia de zirconio, mediante el análisis de los resultados de difracción de rayos $\mathrm{X}$ no se evidenció con claridad fases formadas a partir de este elemento. Badeleyita (ZrO2-monoclinica) aparentemente está presente en las muestras cocidas, acorde con el seguimiento del mayor plano de reflexión de esta fase (111) mostrado en la Figura 4c. El tratamiento Si10-Ti20-Zr70-4 min muestra un ligero incremento de la reflexión del plano anteriormente mencionado.

Espectroscopia de infrarrojos también fue usada para corroborar la presencia de fases de titanio y zirconio. Debido a la gran cantidad de fases presentes y al efecto de sobreposición de las bandas de vibración de los enlaces se realizó el análisis empleando el perfil de segunda derivada de los espectros, esta modificación permitió reducir el efecto de sobreposición. La comparación de las bandas obtenidas con referentes de la literatura [20-22], solo permitió identificar la presencia de bandas de vibraciones para la fase rutilo. En la Figura 5 se presenta las variaciones en la banda de estiramiento del grupo Ti-O en $665 \mathrm{~cm}-1$ del rutilo para los tratamientos realizados.
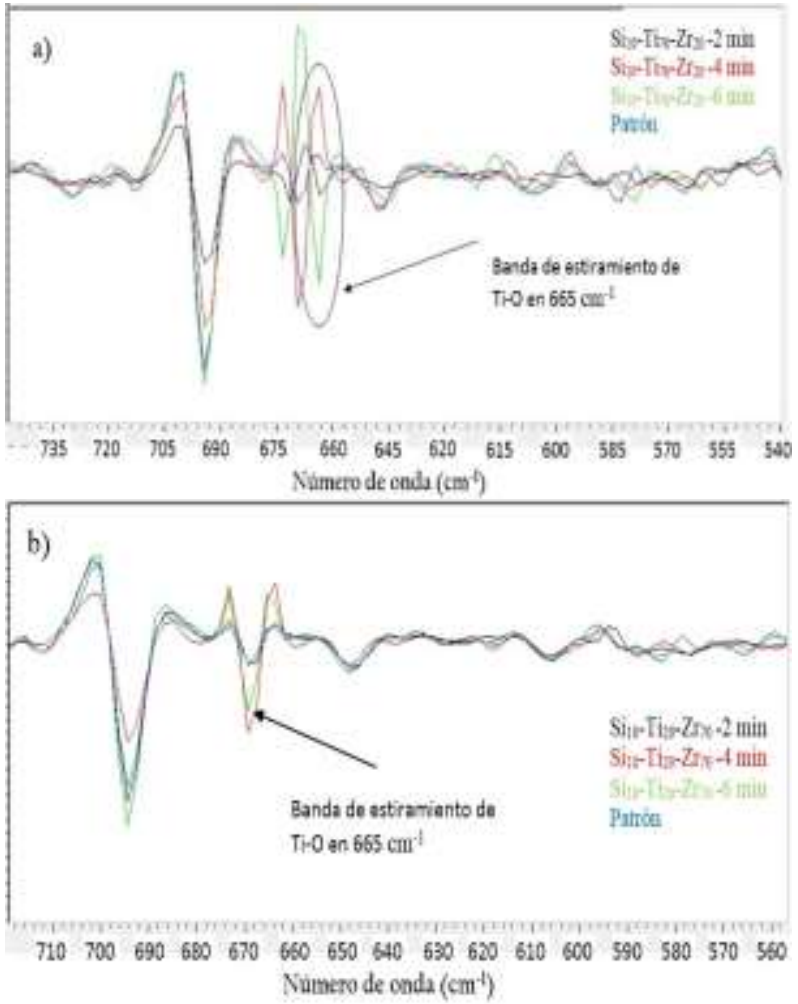

Figura 5. Identificación de la vibración de estiramiento del conjunto Ti-O en los perfiles de segunda derivada de espectros FTIR de las muestras inmersas en el sistema $\mathrm{SiO} 2$ - TiO2 $\mathrm{ZrO} 2$ después del proceso de cocción a $600{ }^{\circ} \mathrm{C}$.
De la Figura 5 se aprecia que para la banda de estiramiento del grupo Ti-O hay un incremento en la intensidad de la señal de dicha banda para las probetas que fueron sometidas al proceso de inmersión en los dos tipos de soluciones. En ambos casos se evidencia que los tratamientos de 4 y 6 minutos son los que presentan una mayor intensidad de la señal, indicando la mayor presencia del enlace y por ende de la presencia del rutilo. Estos resultados obtenidos mediante FTIR presentan correlación con el análisis realizado mediante DRX.

\subsection{Aspectos microquímicos}

Análisis por EDX fueron realizados a fin de complementar las proposiciones establecidas mediante DRX y FTIR. En la Tabla 1 se presenta los resultados obtenidos para todos los materiales.

De los resultados de la Tabla 1 se puede observar como varia la concentración tanto de titanio como de zirconio en los tratamientos de inmersión realizados. Para el caso del titanio se podría pensar que existe una influencia importante del tiempo de inmersión y de la concentración del precursor del óxido de titanio. Aparentemente en concentraciones bajas del precursor de titanio (Si10Ti20-Zr70), el tiempo de inmersión presenta una relación directamente proporcional con la cantidad de titanio retenido en el cerámico, este resultado guarda relación con el análisis de FTIR de la figura 5b para la fase rutilo (TiO2). En altas concentraciones del precursor de titanio (Si10-Ti70-Zr20) se podría pensar que existe un tiempo límite para alcanzar el máximo nivel de retención, en este caso 4 minutos. Los valores obtenidos para el zirconio presentados en la Tabla 1, dejan ver que en altas concentraciones de su precursor (Si10-Ti20-Zr70), el incremento en el tiempo de inmersión no favorece la retención del elemento zirconio en el cerámico. Por otro lado, cuando se usa el precursor pobre zirconio (Si10Ti70-Zr20) se mantiene la misma tendencia de retención del titanio, es decir la influencia del tiempo para una máxima retención.

\subsection{Caracterización físico-cerámica}

Los efectos de los tratamientos de inmersión realizados sobre las propiedades tecnológicas analizadas (Resistencia a la flexión, porcentaje de absorción de agua y densidad aparente) son presentados en las Figuras 6, 7 y 8 .

La Figura 6 muestra la variación del módulo de rotura (resistencia a la flexión) para los diferentes tratamientos después del proceso de cocción a $600{ }^{\circ} \mathrm{C}$. Según lo 
observado en la Figura, los resultados del módulo de rotura de las muestras con el tratamiento Si10-Ti70-Zr20 en todos los tiempos de inmersión, presentan un incremento en el valor de este parámetro comparado con la muestra patrón.
Para el caso de la muestra Si10-Ti70- Zr20 -4 min se presentó un incremento de $20,2 \%$, que representa una mayor resistencia mecánica del material, resultado positivo acorde con el objetivo de investigación propuesto.

Tabla 1. Microanálisis químico de las muestras cocidas (EDX).

\begin{tabular}{|c|c|c|c|c|c|c|c|}
\hline \multirow{4}{*}{ Elemento } & \multicolumn{7}{|c|}{ Tratamiento } \\
\hline & \multicolumn{3}{|c|}{ Sol $\mathbf{S i}_{10}-\mathbf{T i}_{70}-\mathbf{Z r}_{20}$} & \multicolumn{3}{|c|}{ Sol $\mathbf{S i}_{10}-\mathbf{T i}_{20}-\mathbf{Z r}_{70}$} & \multirow{2}{*}{$\begin{array}{c}\text { Patrón } \\
\text { 0 minutos } \\
\end{array}$} \\
\hline & 2 minutos & 4 minutos & 6 minutos & 2 minutos & 4 minutos & 6 minutos & \\
\hline & \multicolumn{7}{|c|}{$\%$ peso } \\
\hline $\mathrm{C}$ & 7,33 & 7,25 & 11,85 & 13,16 & 10,56 & 7,08 & 17,10 \\
\hline $\mathrm{O}$ & 35,34 & 36,65 & 47,20 & 38,01 & 33,71 & 36,23 & 30,53 \\
\hline $\mathrm{Na}$ & 0,68 & 0,61 & 0,68 & 0,55 & 0,63 & 0,66 & 0,63 \\
\hline $\mathrm{Mg}$ & 0,63 & 0,51 & 0,49 & 0,61 & 0,54 & 0,51 & 0,65 \\
\hline $\mathrm{Al}$ & 13,06 & 11,74 & 10,22 & 8,77 & 11,31 & 12,48 & 11,59 \\
\hline $\mathrm{Si}$ & 28,93 & 30,81 & 22,87 & 18,34 & 27,41 & 30,08 & 27,62 \\
\hline $\mathrm{Zr}$ & 0,60 & 0,89 & 0,21 & 3,08 & 2,05 & 2,01 & 0,22 \\
\hline $\mathrm{S}$ & 0,95 & 0,34 & 0,47 & 0,61 & 0,53 & 0,32 & 0,77 \\
\hline $\mathrm{K}$ & 2,02 & 1,96 & 1,08 & 1,07 & 1,55 & 2,28 & 1,64 \\
\hline $\mathrm{Ca}$ & 4,85 & 3,22 & 2,59 & 12,26 & 6,83 & 3,26 & 4,64 \\
\hline $\mathrm{Ti}$ & 1,59 & 1,78 & 0,97 & 0,86 & 0,91 & 1,02 & 0,56 \\
\hline $\mathrm{Fe}$ & 4,01 & 4,25 & 1,37 & 2,68 & 3,98 & 4,06 & 3,99 \\
\hline
\end{tabular}

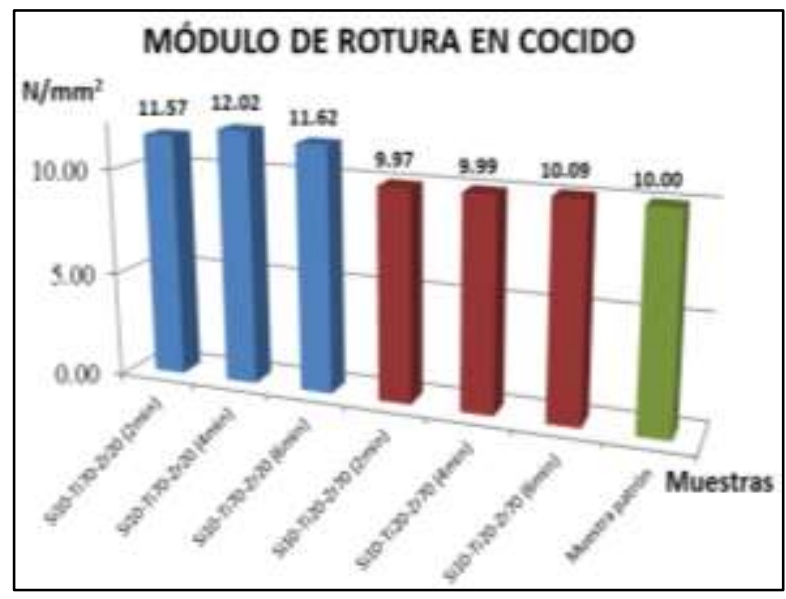

Figura 6. Módulo de rotura (resistencia mecánica a la flexión) de las muestras analizadas.

La densidad aparente es una propiedad que se ve afectada por la porosidad y por el tipo de elementos químicos presentes en la muestra. Como se puede apreciar en la Figura 8 , es evidente que no hay diferencias significativas entre los resultados obtenidos. En el caso del tratamiento con la solución del sistema Si10-Ti70Zr20, la reducción de la porosidad (acorde con el resultado de \% absorción de agua) y la adición de titanio (con masa atómica de 47,8 $\mathrm{g} / \mathrm{mol}$ ) al parecer no son suficientes para lograr un cambio significativo en la densidad aparente de las muestras cerámicas producidas

Este efecto obtenido, podría estar asociado a la disminución de la porosidad y por ende al porcentaje de absorción de agua de las muestras tal como se evidencia en la Figura 7. Se aprecia que las muestras inmersas en la solución Si10-Ti70-Zr20 presentan los valores de absorción de agua más bajo (disminución del 5,3\% en el tratamiento Si10-Ti70-Zr20-2 min con respecto al patrón). Este resultado al ser asociado con los análisis de caracterización anteriormente presentados, conlleva a sugerir que una mayor presencia de titanio superficial en las formas identificadas (rutilo), favorece los valores de las propiedades tecnológicas del cerámico producido.

Para el caso de las muestras inmersas en la solución Si10Ti20-Zr70, no se evidencia ninguna diferencia significativa en los resultados del módulo de rotura y absorción de agua en comparación con el patrón (cerámico sin inmersión). Este hecho permite afirmar que este tratamiento no contribuye a mejorar las propiedades cerámicas del material. 


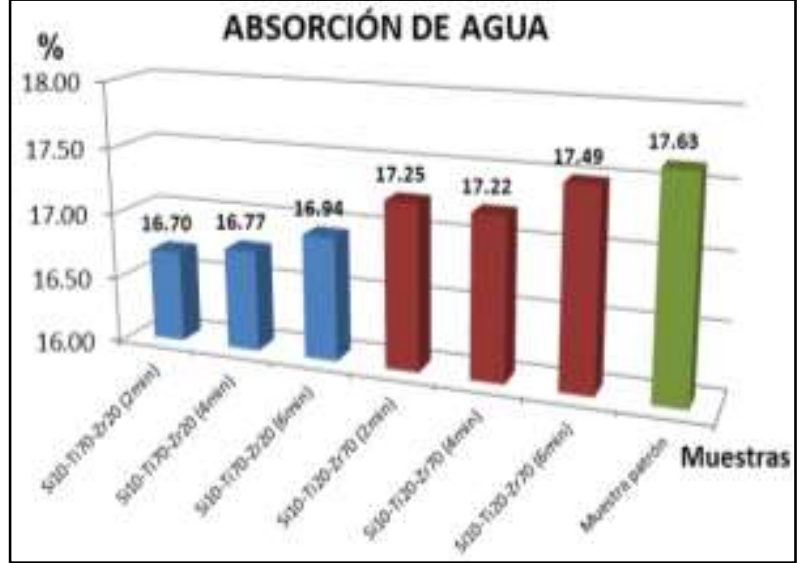

Figura 7. Porcentaje de absorción de agua de las muestras analizadas.

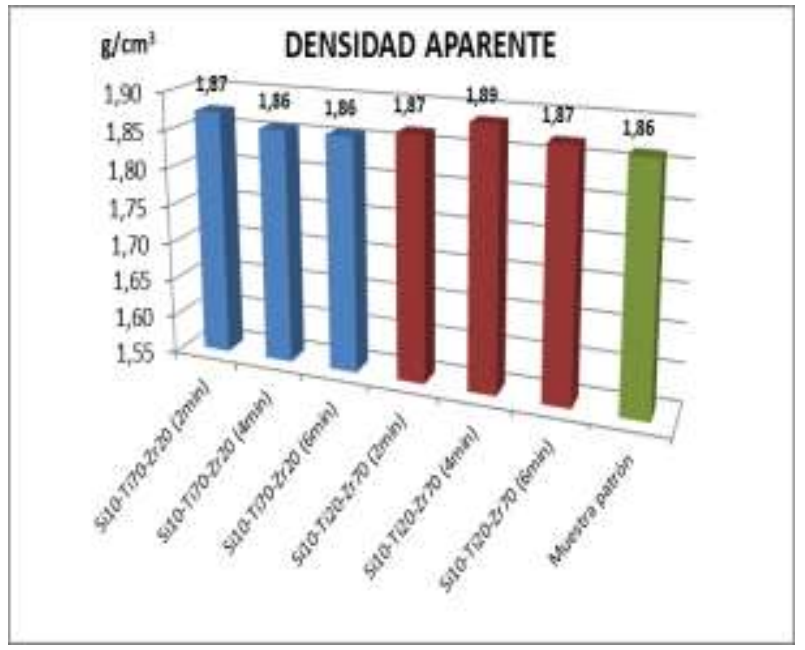

Figura 8. Densidad aparente de las muestras analizadas.

En relación al tratamiento Si10-Ti20-Zr70 y en particular del cerámico que fue sometido a 4 minutos de inmersión (que posee la mayor densidad aparente), el resultado obtenido podría estar asociado a una mayor presencia de zirconio en el material (masa atómica de $91,2 \mathrm{~g} / \mathrm{mol}$ ) acorde con el resultado de la Tabla 1 y no a una mayor densificación por el proceso de cocción.

\section{CONCLUSIONES}

El tratamiento de cocción de la pasta cerámica $\left(1000^{\circ} \mathrm{C}\right)$ permitió la descomposición de las fases arcillosas y de la calcita, sin embargo, no se alcanzó un alto grado de sinterización- vitrificación tal como se manifiesta en los resultados de absorción de agua de la Figura 7. La alta porosidad aun presente en el material, pudo haber jugado un efecto importante en el resultado de la inmersión de las probetas en los soles.
El análisis de difracción de rayos $\mathrm{X}$ de las muestras cocidas (sometidas al proceso de inmersión), permitió inferir la formación de fases como el rutilo y badeleyita, además de un incremento en la concentración de la fase cuarzo de acuerdo con el seguimiento de los principales planos de reflexión. Espectroscopia de infrarrojos permitió corroborar la formación de rutilo en los cerámicos sometidos a inmersión.

El análisis microquímico por EDX permitió tener mayor claridad sobre la cantidad de titanio y zirconio que puede ser retenido en los cerámicos. En el caso del sol Si10Ti70-Zr20, rico en titanio, aparentemente un tiempo de 4 minutos permite tener una mayor concentración de dicho elemento. Para el caso de las muestras inmersas en el sol Si10-Ti20-Zr70 rico en zirconico, tiempos cortos de inmersión aparentemente permiten mejorar la retención del precursor de dicho elemento químico.

En la caracterización física - cerámica se evidenció que las muestras inmersas en la solución Si10-Ti70-Zr20, permiten incrementar la resistencia mecánica de los materiales, siendo el tratamiento de inmersión de 4 minutos el que presentó el mejor comportamiento, con un incremento en el valor del módulo de rotura de $20,2 \%$. Este hecho posiblemente este asociado a la disminución de la porosidad del material y a la mayor presencia de titanio en el cerámico, acorde con los resultados de EDX de la Tabla 1.

No se evidenció variaciones significativas en la densidad aparente de los materiales cocidos, que permita inferir mejoras en la resistencia mecánica de las muestras.

\section{REFERENCIAS}

[1] J. Sánchez., "Estrategias para la competitividad del Clúster de la cerámica del área metropolitana de Cúcuta", Tesis de Maestría, Universidad Nacional experimental del Táchira, San Cristóbal - Venezuela, 2010.

[2] P. Ramirez P., "Planteamiento Estratégico para el Desarrollo de la Innovación Tecnológica en el Sector Cerámico del área metropolitana de Cúcuta”. Tesis de Maestría, Universidad Nacional experimental del Táchira, San Cristóbal - Venezuela, 2010.

[3] Qubit cluster technology based consortium, "Estrategia para la conformación del Clúster y plan estratégico para la cadena de cerámica en Cúcuta", Segundo informe, Cúcuta, 2007.

[4] G. Pinto \& G.Plata., "Desarrollo sostenible en edificaciones". Revista UIS Ingenierías, 9(1),103-121. Jun. 2010. 
[5] I. Nuñez et al., "Investigaciones de interés industrial sobre baldosas cerámicas", [Online] Técnica Industrial, pp. 26, 2004, Disponible en: http://www.tecnicaindustrial.es/tiadmin/numeros/11/38/ a38.pdf

[6] M. Castro, "Recubrimientos protectores obtenidos por deposición electroforética EPD a partir de suspensiones Sol-gel" Tesis de Doctorado en Química, Instituto de Cerámica y Vidrio (CSIC), Madrid, España, 2003

[7] M. Salvador, "Recubrimientos cerámicos obtenidos mediante proyección térmica por plasma atmosférico a partir de polvos reconstituidos Nanoestructurado" [Online], III Congreso Nacional de Pulvimetalurgia, Valencia, 2010, Disponible en: http://digital.csic.es/handle/10261/42686

[8] S. Mollazadeh S, et al., "The role of TiO2, $\mathrm{ZrO} 2, \mathrm{BaO}$ and $\mathrm{SiO} 2$ on the mechanical properties and crystallization behavior of fluorapatite-mullite glassceramics", Journal of Non-CrystallineSolids Volume 361, 1, pp 70, 2013

[9] S. Dudczig et al., "Nano- and micrometre additions of $\mathrm{SiO} 2, \mathrm{ZrO} 2$ and $\mathrm{TiO} 2$ in fine grained alumina refractory ceramics for improved thermal shock performance", Ceramics International, Volume 38, Issue 3, 2012

[10] T. Ebadzadeh, E. Ghasemi, "Effect of TiO2 addition on the stability of $\mathrm{t}-\mathrm{ZrO} 2$ in mullite- $\mathrm{ZrO} 2$ composites prepared from various starting materials". Ceramics International, Volume 28, Issue 4, 447-450, 2002

[11] E. Rambaldi et al., "Nano-oxides to improve the surface properties of ceramic tiles". Boletín de la Sociedad Española de Cerámica y Vidrio. V. 49, 4, 253 258,2010

[12] J. Bautista et al., "Multi-capas cerámicas SiO2TiO2-ZrO2 sintetizadas por el método sol-gel para aplicaciones anticorrosivas", Revista Colombiana de física, Vol. 38, No. 4, 1455-1463, 2006

[13] S. Young et al. "Fabrication and characterization of zirconium carbide $(\mathrm{ZrC})$ nanofibers with termal storage property". Thin solid films. Volume 517, issue 24, 65316538, 2009

[14] J. Bautista, J. Sandoval and C. Ortiz, "Characterizations of coatings obtained by dip coating from sol-gel suspensions". Revista mexicana de física, 55 (1), 144-147, 2009
[15] J. Bautista, C. Ortiz, E. Vera, "Influencia del tipo de sinterizado en el comportamiento anticorrosivo de recubrimientos", Revista Respuestas, 13(2), 5-10, 2008

[16] J. Bautista, "Producción y caracterización de placas cerámicas $\mathrm{SiO} 2$ - TiO2 - ZrO2 sintetizadas por el método Sol gel para aplicaciones anticorrosivas", Tesis de Maestría, UPTC, Colombia, 2010

[17] Instituto Colombiano de Normas Técnicas y Certificación, NTC 4321-4.Baldosas cerámicas. parte 4. Método de ensayo para determinar el módulo de rotura y la resistencia a la flexión. Bogotá D.C. ICONTEC. 2005

[18] Instituto Colombiano de Normas Técnicas y Certificación, Baldosas cerámicas. parte 3. Método de ensayo para determinar la absorción de agua, porosidad aparente, densidad relativa aparente y densidad aparente., NTC 4321-3, 2005.

[19] W. Smykatz., Differential thermal analysis, application and results in mineralogy, Berlin:SpringerVerlag, 1974.

[20] H Van der Marel and H. Beautelspacher., "Atlas of Infrared Spectroscopy of clay minerals and their admixtures". Amsterdam: Elsevier Sci. Pub., B.V., 398. 1976.

[21] N.V. Chukanov., "Infrared spectra of mineral species: extended library". Springer Science \& Business Media. 2013.

[22] Y. Liu et al., "Evolution mechanism of alumina coating layer on rutile $\mathrm{TiO} 2$ powders and the pigmentary properties". Applied Surface Science, 255(16), 74277433. 2009. 